\title{
Lehre an Schulen und Hochschulen in Zeiten von Corona - Ein Erfahrungsbericht aus Sicht der Physikdidaktik
}

\author{
K. Falconer, S. Hoffmann, A. Schadschneider ${ }^{*}$ \\ Institut für Physikdidaktik, Mathematisch-Naturwissenschaftliche Fakultät, Universität zu Köln
}

\begin{abstract}
Die Einschränkungen in der Präsenzlehre sind insbesondere für experimentelle Wissenschaften wie die Physik eine besondere Herausforderung. In diesem Beitrag wollen wir einige Hilfsmittel und Konzepte vorstellen, die sich in der Ausbildung von angehenden Physiklehrer*innen bewährt haben. Wir werden getrennt auf die verschiedenen Veranstaltungsformen (Vorlesungen, Seminare, Praktika) eingehen und diskutieren, wie sie in einem Online-Format umgesetzt werden können. Tools wie Zoom, llias, Mural etc. können dabei insbesondere kooperative Lernformen unterstützen, die in der Online-Lehre besonders nützlich sind, um ein Mindestmaß an sozialer Interaktion zu gewährleisten. Außerdem stellen wir Konzepte vor, die zu einer reflektierteren Auseinandersetzung mit den Inhalten der Veranstaltung führen. Schließlich werden die Probleme bei Online-Prüfungen diskutiert, bei denen ein Kompromiss zwischen bestmöglicher Überwachung und der Wahrung der Privatsphäre gefunden werden muss. Hier schlagen wir mögliche Lösungen vor, die sich bereits in der Praxis bewährt haben.
\end{abstract}

The limitations of face-to-face teaching are a particular challenge for experimental sciences such as physics. In this paper, we will present some tools and methods that have proven successful in the preparation of prospective physics teachers. We will look separately at the different types of courses (lectures, seminars, practical labs) and discuss how they can be implemented in an online format. Tools such as Zoom, Ilias, Mural etc. can support cooperative learning groups in particular, which are especially useful in online teaching to ensure a minimum of social interaction. We also introduce methods that lead to students' more reflective engagement with the content of the course. Finally, we discuss the problems of online examinations, where a compromise has to be found between the best possible supervision and the preservation of privacy. Here we suggest possible solutions that have already proven themselves in practice. 


\section{Einleitung}

Die Notwendigkeit zur Online-Lehre stellt Schule und Hochschule vor enorme Herausforderungen. Gleichzeitig bietet sie (nicht nur) für Didaktiker die Chance, Ideen und Konzepte in einem großen Maßstab zu testen. In diesem Beitrag soll an einigen Beispielen aus diesem großen Experiment diskutiert werden, welche Chancen aber auch Risiken dies birgt.

Eine wichtige Frage ist die nach der geeigneten Veranstaltungsform. Vorlesungen lassen sich nicht 1-zu-1 in ein Online-Format übersetzen. Welche Möglichkeiten gibt es hier? Und wie kann man die Studierenden bzw. Schüler*innen aktivieren?

Besondere Herausforderungen bestehen für die experimentellen Wissenschaften, insbesondere wenn durch die Randbedingungen Präsenz in Laboren nicht möglich ist. Hier sind neue Ideen gefragt, wenn man diese Praktika interessant, lehrreich und sicher durchführen möchte.

Schließlich stellt sich die Frage nach der Zukunft der Lehre in der Post-Corona-Zeit! Wird wieder alles wie vorher? Gibt es Neuerungen, die sich bewährt haben und die auch nach Corona den Lehrbetrieb bereichern werden?

\section{Technische Vorbemerkungen}

Der Erfolg von Online-Lehre hängt natürlich wesentlich von den technischen Randbedingungen ab. Auf die damit verbunden Probleme (z.B. Qualität der Internetverbindung, Datenschutz etc.) soll hier nur am Rande eingegangen werden.

Die Universität zu Köln (UzK) hat sich hinsichtlich der Präsenzlehre während der CoronaKrise relativ konservativ positioniert, so dass die Lehre seit März 2020 fast ausschließlich online durchgeführt werden konnte. Um hier die technischen Voraussetzungen für Mitarbeiter und Studierende zu schaffen, hat die UzK 50000 Zoom-Lizenzen erworben. Dadurch konnten mit dem Hersteller günstige Konditionen ausgehandelt werden, gerade im Hinblick auf die Datenschutzbestimmungen. So werden die Daten nur auf Servern innerhalb der EU verarbeitet.
Ein weiterer Grund für die Entscheidung für Zoom waren die guten Erfahrungen, die am Institut für Physikdidaktik bereits seit mehreren Jahren gemacht wurden. Dadurch konnten Mitarbeiter*innen auch als Ausbilder*innen für universitätsinterne Fortbildungen in der Zoom-Nutzung eingesetzt werden.

Bereits vor der pandemiebedingten Aussetzung der Präsenzlehre haben wir mobile Whiteboards (ca. 80 × $60 \mathrm{~cm}$ große kunststoffbeschichtete und mit Filzschreibern beschreibbare Holztafeln) für Gruppenarbeiten in Seminaren, Vorlesungen und Laboren für formatives Assessment verwendet. Formatives Assessment ist die kontinuierliche Überprüfung und Erhebung des Wissensstandes des Lernenden im Lernprozess. Für den Dozenten bietet es eine Möglichkeit, den Stand des Verständnisses der Studenten für die Materialien zu ermitteln. Gleichzeitig ist es ein Werkzeug, um die Beurteilung des eigenen Unterrichts- und Lehrplanmaterials zu erleichtern. Die formative Bewertung besteht also nicht nur darin, zu prüfen, ob die Studierenden richtig liegen, sondern soll gleichzeitig die zugrunde liegenden Vorstellungen und Kompetenzen der Studierenden untersuchen. Eine sehr anschauliche Einführung und Diskussion der Vor- und Nachteile des formativen Assessments findet man in [1].

Mit den oben angesprochenen Whiteboards konnten Dozenten auf einen Blick sehen, welches Verständnis die Studierenden entwickelt hatten. Nach der Implementierung von Online-Kursen haben wir versucht, die in Zoom integrierten Whiteboards zu verwenden. Während die Whiteboards für Diskussionszwecke nützlich waren, war es nicht einfach, alle Boards auf einen Blick zu sehen und Boards zu organisieren und dokumentieren. Nach der Erprobung verschiedener Alternativen wählten wir Mural [2] als Ersatz für die physischen Whiteboards aus. Wir haben festgestellt, dass die großen Vorlesungen in Zoom Break Out Rooms (BORs) unterteilt werden können und dass der Dozent mit Hilfe von vervielfältigten Gruppen-Murals in Echtzeit sehen kann, woran die Studierenden arbeiten und welches Verständnis sie erzielen können. Dies ermöglicht auch ein einfaches Teilen von Arbeitsergebnissen durch Studierende in Seminaren, Vorlesungen und 
Praktika. Der Echtzeit-Modus erlaubt es, alle Aktivitäten bis hin zu Cursor-/Mausbewegungen aller Studierenden und Lehrenden einzublenden. Dies ist allerdings für alle Beteiligten mit größerer Rechenleistung im Browser auf dem Computer oder auf der Tablet-App verbunden. Dieser Modus kann allerdings deaktiviert werden.

Ein weiteres Tool, das seit vielen Jahren standardmäßig in der Lehre eingesetzt wird, ist die Lernplattform Ilias (Integriertes Lern-, Informations- und Arbeitskooperations-System) [3], mit der internetbasierte Lehr- und
Lernmaterialien erstellt und verfügbar gemacht werden können. Sie bietet vielfältige Möglichkeiten von der Planung von Veranstaltungen bis hin zur Lernfortschrittskontrolle mit verschiedenen Testformaten. AuBerdem wird die Kommunikation mit den Studierenden über E-Mail, Chats oder Foren unterstützt. Diese Plattform ist den Studierenden sehr vertraut, was den Übergang zu Online-Formaten deutlich erleichtert hat.



Abb. 1: Einsatz von Murals in einem Didaktik-Seminar: Studierende entwickeln in Gruppen ein Verständnis für einfache elektrische Schaltungen. Die Abbildung zeigt die Ansicht des Lehrenden. Jedes Feld stellt das Mural einer Gruppe (bzw. eines Breakout-Rooms) dar. Die Lehrenden haben daher den Überblick über die Aktivitäten aller Gruppen und können ggfs. unterstützend eingreifen. Ein vergrößerter Ausschnitt ist in Abb. 2 dargestellt.

\section{Vorlesungen}

Vorlesungen sind das zentrale Veranstaltungsformat an Hochschulen. Für die Realisierung im Rahmen der Online-Lehre gibt es zahlreiche Optionen. Die einfachste Möglichkeit besteht natürlich darin, so wenig wie möglich zu ändern. Die Vorlesung findet dann live via Zoom statt, wobei die Dozierenden wahlweise in einem Hörsaal vor einer Tafel stehen oder vom Home Office aus z.B. mit einer Powerpoint-Präsentation arbeiten. Der offensichtliche Vorteil ist, dass hier (außer den technischen Randbedingungen) nicht viel gegenüber den klassischen Vorlesungen verändert werden muss. Nachteilig wirkt sich aber aus, dass die Kommunikation zwischen den Dozenten und den Studierenden noch schwieriger wird. Aus Sicht der Dozierenden fehlt vor allem das unmittelbare Feedback über Mimik oder Gestik. Daher lässt es sich nur sehr schwer abschätzen, wie gut die Vor- 
lesung "ankommt". Dies wird weiter durch die Tatsache verstärkt, dass die meisten Zuhörer*innen die Kameras abschalten und man daher in Zoom nur auf schwarze Kacheln schaut. Es fehlt jegliches Feedback von der Zuhörerschaft. Fragen werden kaum gestellt, da dies den Vortragsfluss unterbricht. Die Funktionen in Zoom („Hand heben“) sind hier keine wirkliche Hilfe, da man diese oft nicht im Auge behalten kann.

Ein erster Schritt weg von der Live-Vorlesung über das Internet sind Vorlesungsaufzeichnungen. In der einfachsten Varianten sind es im wesentlichen Live-Vorlesungen, die aufgezeichnet und idealerweise noch editiert werden. Aus didaktischer Sicht sollte die Länge der üblichen Aufmerksamkeitsspanne angepasst sein. Mehrere kurze Videos, idealerweise mit einem thematisch abgeschlossenen Inhalt, sind effektiver als eine 90-minütige Aufzeichnung. Dieser Effekt wird noch verstärkt, wenn sich an ein solches kurzes Video eine angepasste Übung anschließt, die das Verständnis weiter vertieft.

Vorlesungsvideos sind in Befragungen von den Studierenden sehr gut bewertet worden. Als Hauptvorteil wird dabei ein individuelles Lerntempo angesehen. So lassen sich Teile der Vorlesung leicht öfter ansehen, was besonders bei sehr "dichten" Präsentationen wichtig ist. Ein großes Problem, gerade bei den Live-Vorlesungen, ist aber die mangelnde Aktivierung der Studierenden. Hier sollten immer wieder Phasen eingebaut werden, in denen sie selbst aktiv werden müssen, sei es in Diskussionsrunden oder mit kleineren Aufgaben.

Eine vollkommen andere Herangehensweise ist der flipped oder inverted classroom. Hierbei werden die klassischen Rollen von (Präsenz)Vorlesung und selbstständig zu bearbeitenden Übungen (in der Regel als Hausaufgaben) vertauscht. Die Inhalte der Vorlesung werden z.B. über ein Skript zur Verfügung gestellt. Die Zeit, in der üblicherweise die Vorlesung stattgefunden hätte, wird dann für Fragen, Diskussion und Bearbeitung von Übungsaufgaben genutzt. Dies erleichtert das Erkennen von grundlegenden Verständnisproblemen. Das Konzept lässt sich auch sehr gut mit Vorlesungsaufzeichnungen kombinieren.
Wie kann man gewährleisten, dass die Studierenden regelmäßig das Skript bzw. die Vorlesungsvideos ansehen? Als ein geeignetes Mittel haben sich hier Tests erwiesen, die sich z.B. in Ilias einfach realisieren lassen. Ilias bietet eine Testumgebung mit unterschiedlichen Fragentypen an, z.B. Single- oder Multiple-Choice, Zuordnungs-, Lückentextfragen oder auch Fragen mit Freitextantworten. Die Testauswertung geschieht automatisch (was aber bei Freitextantworten eher nicht zu empfehlen ist). Daher erhalten die Studierenden unmittelbar nach Testende ein Feedback.

Solche Tests lassen sich sehr gut zur Inhaltssicherung einsetzen. So gibt es zu jedem Kapitel des Skripts (mindestens) einen Test, wobei sich die Fragen alleine mit dem Skript beantworten lassen. Im Schnitt sollten hier 1-2 Fragen pro Seite des Skriptes gestellt werden. Durch eine Deadline für die Bearbeitung lässt sich sicherstellen, dass die Studierenden „am Ball bleiben" und nicht den Anschluss an die Vorlesung verlieren. Außerdem sollen die Fragen der Orientierung der Studierenden dienen. Anhand von Skripten oder Büchern ist es für Anfänger oft sehr schwer erkennbar, was wirklich wichtig und was eher Beiwerk ist. Dies lässt sich durch geeignete Fragen etwas besser steuern. Für die Vergabe der Credit Points ist dann u.a. das Bestehen aller Tests ein Kriterium. Durch eine hohe Mindestpunktzahl (mindestens 75\%) für das Bestehen kann man auch dafür sorgen, dass dieser nicht „zufällig“ bestanden werden kann. Dies sollte aber durch die Möglichkeit, einen Test (mehrfach) zu wiederholen, flankiert werden.

Die Erfahrung hat gezeigt, dass sich die Studierenden - trotz bestandener Tests - nur oberflächlich mit den vorzubereitenden Inhalten auseinandergesetzt haben. Oftmals wird gerade genug getan, um den Test bestehen zu können. Daher haben wir im zweiten Online-Semester diese Tests durch ein neues Hilfsmittel ersetzt bzw. ergänzt, das eine reflektierte Auseinandersetzung mit den Inhalten fördert. Die Studierenden mussten dabei eine Art Logbuch (Reading Log) [4,5] führen, bei dem u.a. gewisse Leitfragen zu beantworten waren. In der ersten Version wurde hierzu Reading Logs, die bereits seit Jahren erfolgreich an amerikanischen Hochschulen 
eingesetzt werden, an die Gegebenheiten einer deutschen Hochschule angepasst.

Die Studierenden sollen den vorzubereitenden Teil des Skripts (mindestens) zweimal lesen. Nach dem ersten Lesedurchgang sollen dann die wesentlichen Inhalte in sehr kurzer Form stichwortartig oder durch Skizzen etc. zusammengefasst werden. Außerdem werden in einem Glossar die neu aufgetauchten Begriffe und Größen und ihre Definition oder eine Beschreibung zusammengefasst. Um zu einer reflektierteren Beschäftigung mit den Inhalten zu gelangen, sollen außerdem offene Fragen notiert werden, die sich beim Lesen ergeben haben. Dies soll den Blickwinkel für den zweiten Lesedurchlauf fokussieren. Nach dem zweiten Lesen sollen dann noch einmal die drei wichtigsten neuen Ideen aufgeführt werden. Weiterhin soll das neue Wissen in einen größeren Kontext eingeordnet werden, z.B. über mögliche Anwendungen oder bereits bekannte Ergebnisse aus anderen Bereichen. Abschließend sollen die Studierenden kurz diskutieren, welche neuen Einsichten sich durch das nochmalige Lesen ergeben haben und ob weiterführende Fragen bestehen, die man in der Vorlesung diskutieren sollte. Außerdem soll noch die für das Lesen und Ausfüllen des Logbuchs benötigte Zeit notiert werden.

Die Logbücher sind am Tag vor der Vorlesung über Ilias einzureichen. Die Studierenden erhalten (in der Regel) noch vor der Veranstaltung ein Feedback zu ihrem Logbuch. Im Sinne des Just-in-Time-Teaching [6] wird in der Vorlesung auf die eingereichten Logbücher eingegangen. Man erhält durch dieses Vorgehen einen sehr guten Überblick darüber, ob die Studierenden wirklich die wesentlichen Inhalte erkannt und verstanden haben. Es wird vor allem versucht, auf die Fragen einzugehen, um die Sinnhaftigkeit der Logbücher zu illustrieren.

\section{4. Übungen und Praktika}

Übungen leben i.a. sehr stark von den Interaktionen der Studierenden untereinander und mit dem Übungsleiter bzw. Betreuer. Dies kann online nur schwer erreicht werden. Eine Möglichkeit hierzu ist die Arbeit in Kleingruppen, z.B. in Breakout Rooms (BORs) innerhalb von Zoom Meetings. Die Betreuer wechseln dabei regelmäßig zwischen den Räumen um Fragen zu beantworten und zu sicherzustellen, dass zielgerichtet gearbeitet wird. Gerade in den Lehramtsstudiengängen hat es sich bewährt, dass dabei komplementäre Aufgaben in den BORs bearbeitet werden. Alle Studierenden kommen dann immer wieder in der Hauptsitzung zusammen, um ihre Ergebnisse vorzustellen und zu diskutieren. Dies führt auch zur notwendigen Abwechslung und verbessert die Aufmerksamkeit, da immer wieder neue Impulse gegeben werden.

Übungselemente können auch direkt in Vorlesungen eingebaut werden. Dies liefert eine unmittelbare Rückmeldung über mögliche Verständnisprobleme. Gleichzeitig werden die Studierenden aktiviert und bleiben nicht nur passiv in ihrer Rolle als Zuhörer. Z.B. können Multiple Choice Fragen für ein formatives Assessment in Präsenzveranstaltungen benutzt werden. Hierzu haben wir auch schon früher in Präsenzveranstaltungen Software wie ILIAS-Live-Votings, Plickers [7] oder Kahoot [8] eingesetzt. Es gibt bereits zahlreiche Konzept- und Anwendungsfragen in MultiChoice-Formaten (Konzept-Tests [9]).

In den Online-Kursen konnten einige dieser Tools ebenso verwendet werden. Darüber hinaus nutzen wir die Umfrage-Funktion von Zoom (Abb. 2), die den Vorteil hat, dass sie in das Zoom-Userinterface integriert ist und sich automatisch für die Studierenden im Vordergrund öffnet. Nachteil ist allerdings, dass keine Abbildungen in Fragen oder Antworten eingefügt werden können. Der ZoomChat erwies sich ebenso häufig als schnelles Mittel für ein individuelles Feedback.

Die Plattform Mural bietet einen so genannten "Private Mode“, in dem Studierende nicht länger sehen, welche Änderungen andere Lehrende und Studierende vornehmen. Dadurch können individuelle Vorhersagen abgefragt und später diskutiert werden. 

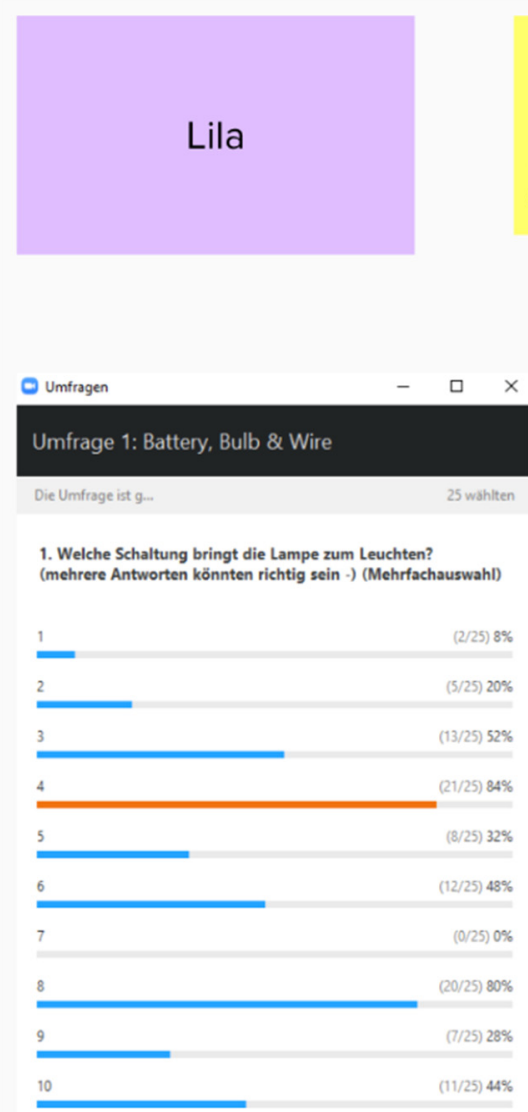

Schaut euch die Ergebnisse an. Versucht als Gruppe zu klären warum die Lampe angeht oder nicht.

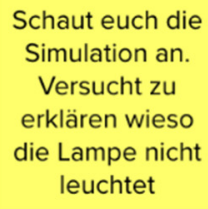

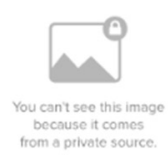

Colorodo

\section{Battery, Bulb and Wire(s).}

Hier siehst du Bilder von möglichen Verbindungen einer Batterie mit einer Glühbirne und keinem, einem oder zwei Kabeln.

Bei welchen Bildern kann die Lampe leuchten und bei welchen nicht? Begründe jeweils!



Abb. 2: Dieser vergrößerte Ausschnitt aus Abb. 1 zeigt die Bearbeitung der Aufgabe "Welche Schaltung bringt die Glühbirne zum Leuchten? Halten Sie Ihre Vorhersagen in Zeichnungen fest und überprüfen diese mit der PhET-Simulation "Stromkreise schalten: Gleichstrom" [10]." durch eine Studierendengruppe in einem Mural. Die hervorgehobene Abbildung zeigt Details der Bearbeitung einer Gruppe, z.B. auf virtuellen Post-It Zetteln festgehaltene Kommentare und das Ergebnis einer Zoom-Umfrage (links), die als Diskussionsgrundlage dient und das Ergebnis der anschließenden Diskussionen.

Praktika sind vermutlich am stärksten von den Einschränkungen für Präsenzveranstaltungen betroffen. Gerade die notwendigen Erfahrungen im Umgang mit Geräten lassen sich eigentlich nur in Präsenzveranstaltungen vermitteln. Wenn eingeschränkte Präsenz möglich ist, kann man sich mit verschiedenen Notlösungen behelfen. Z.B. ist eine Art "Schichtbetrieb" möglich, bei der die Studierenden einzeln statt in Kleingruppen Versuche durchführen. Dies ist allerdings mit sehr starken Belastungen für die Betreuer verbunden. Daher wurden zum Teil Versuche mit einem Gruppenmitglied im Labor und 2-3 weiteren, über Zoom zugeschalteten Studierenden, durchgeführt. Hier muss man allerdings dafür sorgen, dass diese genügend sinnvolle Tätigkeiten zur Unterstützung haben und nicht nur Zuschauer sind. Die Auswertung der Versuchsergebnisse geschieht dann wie im Präsenzbetrieb.

Eine alternative Herangehensweise versucht die Kreativität der Studierenden zu nutzen und sie in eine geeignete Bahn zu lenken. Man kann z.B. relativ allgemeine Aufgaben vergeben, die dann mit den jeweils zur Verfügung stehenden Mitteln (z.B. im Haushalt) zu Iösen sind. Die Studierenden müssen dann die Experimente selbst konzipieren statt, wie leider oft in Praktika üblich, an fertig aufgebauten Versuchen zu arbeiten. Bei solchen Experimenten lassen sich sehr gut die Möglichkeiten moderner Smartphones oder Tablets nutzen, die mit einer Vielzahl an Sensoren ausgestattet sind. Diese können mit einer Software wie phyphox [11] ausgelesen werden. Mit Apps wie Video Physics [12] lassen sich Bewegungen filmen und auswerten. Dies sind nur zwei Beispiele für die Möglichkeiten, 
die die Verwendung von Smartphones bietet. Gerade für Schüler*innen ist das sehr attraktiv, da man damit Beispiele aus der unmittelbaren Lebenswelt bearbeiten kann.

Eine weitere Möglichkeit, die aber in der Regel eher eine Ergänzung denn Ersatz für reale Versuche sein wird, ist die Verwendung von Simulationen. Für die Naturwissenschaften, insbesondere die Physik, ist hier PhET [13] sehr empfehlenswert. Diese Plattform wurde vor fast 20 Jahren vom Physiknobelpreisträger Carl Wieman gegründet und umfasst mittlerweile mehr als 150 Simulationen mit fast 3000 Anwendungsbeispielen von Praktikern aus Schule und Hochschule. Neben Simulationen aus dem Bereich der Physik gibt es auch solche zur Chemie, Mathematik, Biologie und den Geowissenschaften. Für die Physikausbildung an der Hochschule sind die Apps zu elektrischen Schaltkreisen oder zur Optik sehr hilfreich, da sie durch ihre Gestaltung konzeptionell sehr nahe an realen Versuchen sind. Man kann "echte" Messungen durchführen, z.B. an einfachen Schaltungen (Abb. 3).

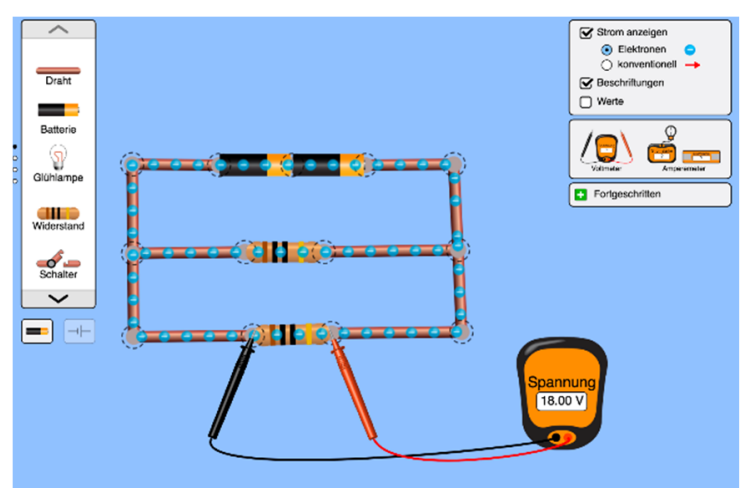

Abb. 3: PhET-App zu Gleichstromkreisen [10]

Eine Alternative, die für Studierende sehr attraktiv ist, ist das Arbeiten mit programmierbaren Robotern. Hier haben wir gute Erfahrungen mit den Robotern ("Codey Rocky") von Makeblock [14] gemacht (Abb. 4). Diese lassen sich mit der einfachen Programmiersprache Scratch [15], die am MIT speziell für jüngere Kinder entwickelt wurde, programmieren. Die Roboter lassen sich mit zahlreichen Sensoren ausstatten und ermöglichen so die Durchführung von physikalischen Projekten. Ein konkretes Beispiel werden wir in Abschnitt 6 vorstellen.

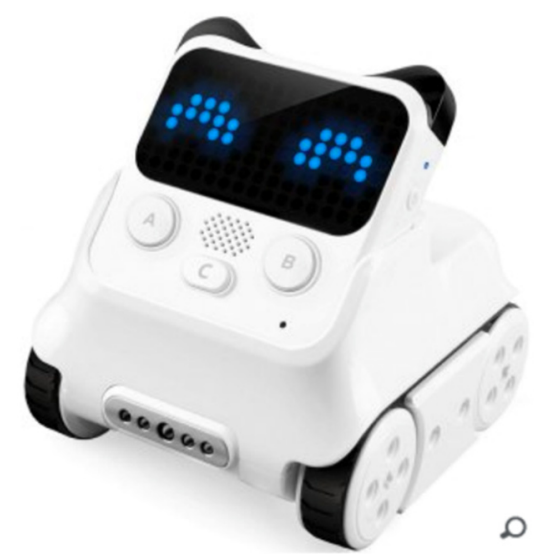

Abb. 4: Ein programmierbarer CodeyRocky Roboter von Makeblock. Die Roboter sind beweglich und können mit verschiedenen Sensoren ausgestattet werden.

\section{Prüfungen}

Zum Studium gehören immer auch Prüfungen. Um größere Verzögerung zu vermeiden, ist es natürlich nicht möglich, ein Jahr oder mehr auf jegliche Prüfungstätigkeit zu verzichten.

Gute Erfahrungen haben alle Beteiligten mit mündlichen Prüfungen via Zoom (und anderen Videokonferenzsystemen) gemacht. Der Ablauf ist hier relativ ähnlich zu dem einer Präsenzprüfung. Plattformen wie llias stellen oft Möglichkeiten für elektronische Tests zur Verfügung (siehe oben im Abschnitt "Vorlesungen"), in der Regel mit der Option für eine automatische Auswertung von Multiple Choice Tests. Dies erscheint auf den ersten Blick sehr praktisch, ist aber mit zahlreichen Gefahren verbunden. Die Qualität solcher Tests für die Leistungsüberprüfung steht und fällt mit der Qualität der Fragen und insbesondere der Distraktoren, also der angebotenen falschen Antworten. Um einen guten und aussagekräftigen Multiple Choice Test zu erstellen, muss man bereits eine sehr gute Vorstellung von den Präkonzepten („Fehlvorstellungen") der Studierenden haben. Man muss also die möglichen Fehler antizipieren und die Antworten dementsprechend wählen. In vielen Bereichen der Physik sind Präkonzepte sehr gut untersucht, z.B. in der Mechanik und der Elektrik.

Die Durchführung klassischer Klausuren in Online-Formaten ist zwangsläufig mit großen Problemen behaftet, insbesondere durch 
den Konflikt zwischen der notwendigen Überwachung und dem Schutz der Privatsphäre. Es gibt aber auch hier Möglichkeiten, diesen Spagat zu bewältigen. Dabei ist aber zu beachten, dass die Studierenden die Möglichkeiten moderner Medien sehr gut kennen und für sich zu nutzen wissen. Dies hat in weniger gut konzipierten Online-Klausuren zu groß angelegten Täuschungsversuchen geführt [16]. Solche Täuschungen, z.B. durch Kommunikation der Teilnehmer*innen untereinander (inklusive einer "Aufteilung" der Aufgaben [17]) sind auch in Formaten wie Open-Book Klausuren möglich, bei denen die Nutzung externer Hilfsmittel wie Bücher und/oder Internetquellen erlaubt sind.

In Abstimmung mit dem Justitiariat der Universität zu Köln haben wir ein Format entwickelt, das die Durchführung von Klausuren ermöglicht, bei gleichzeitiger Videoüberwachung der Teilnehmer*innen im Rahmen der rechtlichen Möglichkeiten. Eine solche Überwachung ist möglich, wenn nur eine Kamera verwendet wird und der Bildausschnitt die Privatsphäre wahrt [17]. Zur Kontrolle vor der Prüfung oder in Verdachtsfällen ist auch ein schneller Kameraschwenk durch den Raum erlaubt. Ein illustratives Beispiel ist in Abb. 5 gezeigt. Zur Videoübertragung wird in der Regel ein Smartphone verwendet, das heute praktisch alle Studierenden besitzen. Es wird so angebracht, dass es einen Blick über die Schulter des Klausurteilnehmers erlaubt, wobei stets die Hände und der Bildschirm des Rechners, auf dem Aufgaben zur Verfügung gestellt werden, zu sehen sind. Für die Halterung des Smartphones gibt es viele kreative Ideen, die sich Haushaltsgegenstände zu Nutze machen (Abb. 6).

Zur Durchführung der Klausur werden die Teilnehmer*innen auf BORs verteilt, wobei jedem BOR neben einem Teilnehmer noch eine Aufsichtsperson zugeordnet wird.

Diese Aufteilung erlaubt es, neben dem Video auch den Ton übertragen zu lassen, ohne dass dadurch andere Teilnehmer*innen gestört werden. Dies verhindert die Kommunikation mit anderen Personen, die sich ggfs. im gleichen Raum befinden und unerlaubte Unterstützung geben. Außerdem können leicht Rückfragen gestellt werden. Mikrofon und Lautsprecher auf Seiten der Klausurteilnehmer sind permanent angeschaltet, damit Fragen und Hinweise der Aufsichtspersonen gehört werden. Die Aufsichtspersonen schalten ihre Mikrofone nur bei Bedarf ein, da sie mit mehreren Geräten mehrere BORs gleichzeitig überwachen.

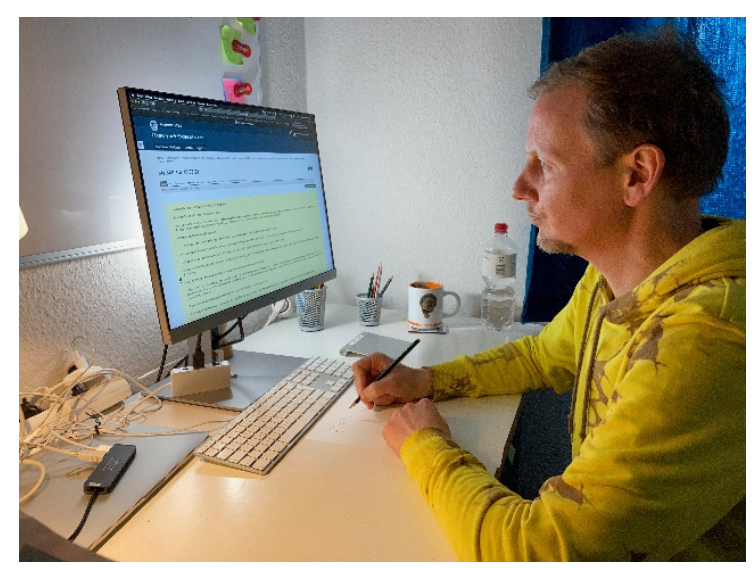

Abb. 5: Erlaubte Form der Videoüberwachung bei Online-Klausuren über die Kamera eines Mobiltelefons.

Natürlich bietet auch dieses Verfahren keine 100\%ige Sicherheit gegenüber Täuschungsversuchen. Das ist aber auch gar nicht wünschenswert! Prinzipiell sollten die OnlineKlausuren eine Sicherheit bieten, die mit der bei den klassischen Präsenzklausuren vergleichbar ist.

Wir haben solche Klausuren im vergangenen Semester mehrfach erfolgreich durchgeführt. Die Studierenden waren dabei sehr kooperativ und zeigten volles Verständnis für die Maßnahmen. Technische Probleme sind dabei nur sehr selten aufgetreten. Sie waren etwa so häufig wie bei klassischen Klausuren, wo z.B. Störungen im Nahverkehr öfter dazu führen, dass Studierende Klausuren verspätet beginnen oder gar ganz versäumen. In den gemäß dem oben beschriebenen Verfahren durchgeführten Klausuren kam es außerdem zu keinen offensichtlichen Täuschungsversuchen. 


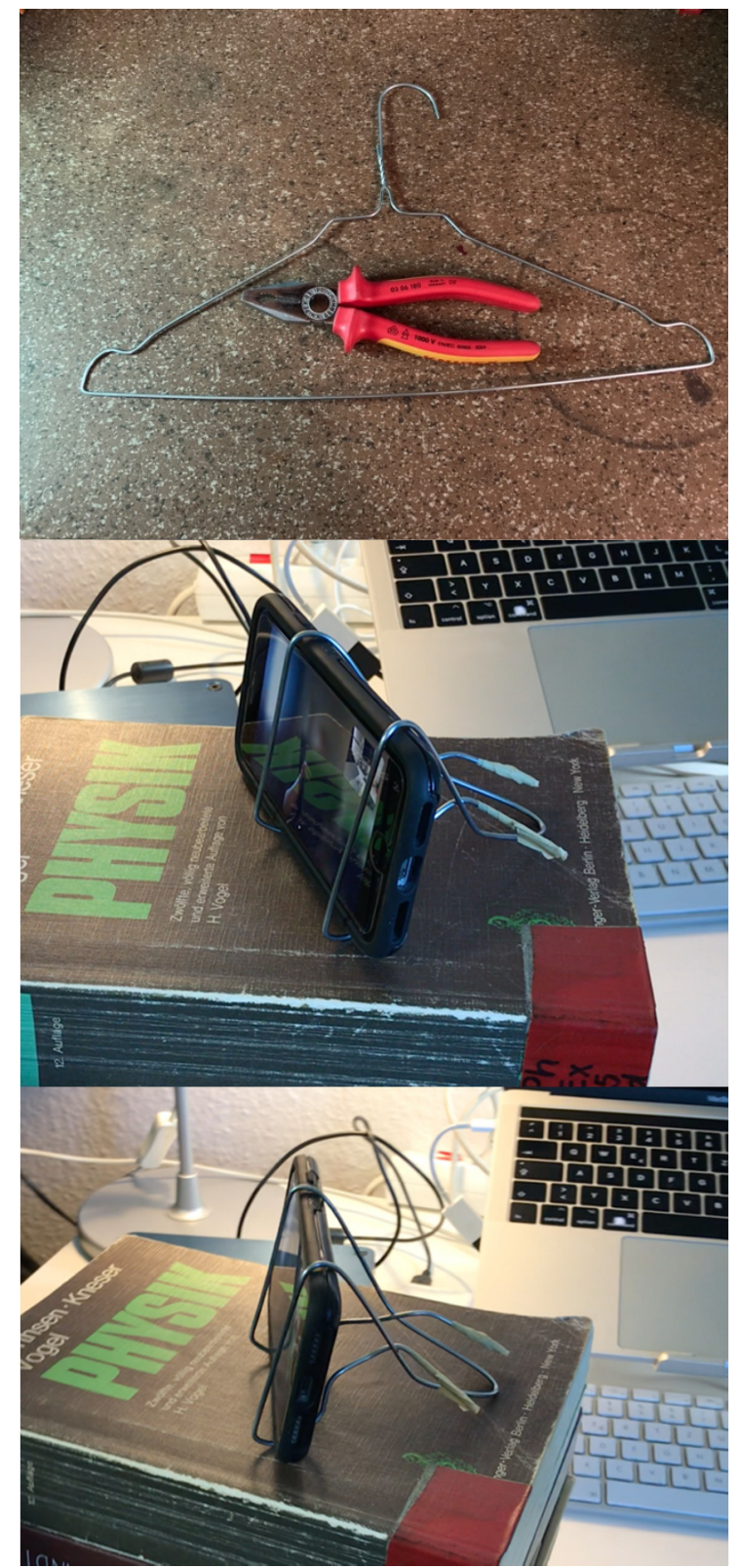

\section{Konkrete Beispiele}

Im Folgenden möchten wir an einigen konkreten Beispielen demonstrieren, wie sich oftmals nicht erwartete positive Ergebnisse durch Online Lehre erzielen lassen.

In der Veranstaltung "Mathematische Methoden“ lernen angehende Physik-Lehrer*innen an Haupt-, Real- und Gesamtschulen das notwendige mathematische Rüstzeug für das Studium. In diesem Fall geht es nur unwesentlich über den üblichen Stoff in einem Mathematik-Leistungskurs hinaus (den aber in der Regel nur ein kleiner Teil der Teilnehmer*innen besucht hat!). Diese Veranstaltung wird schon seit vielen Jahren im Inverted

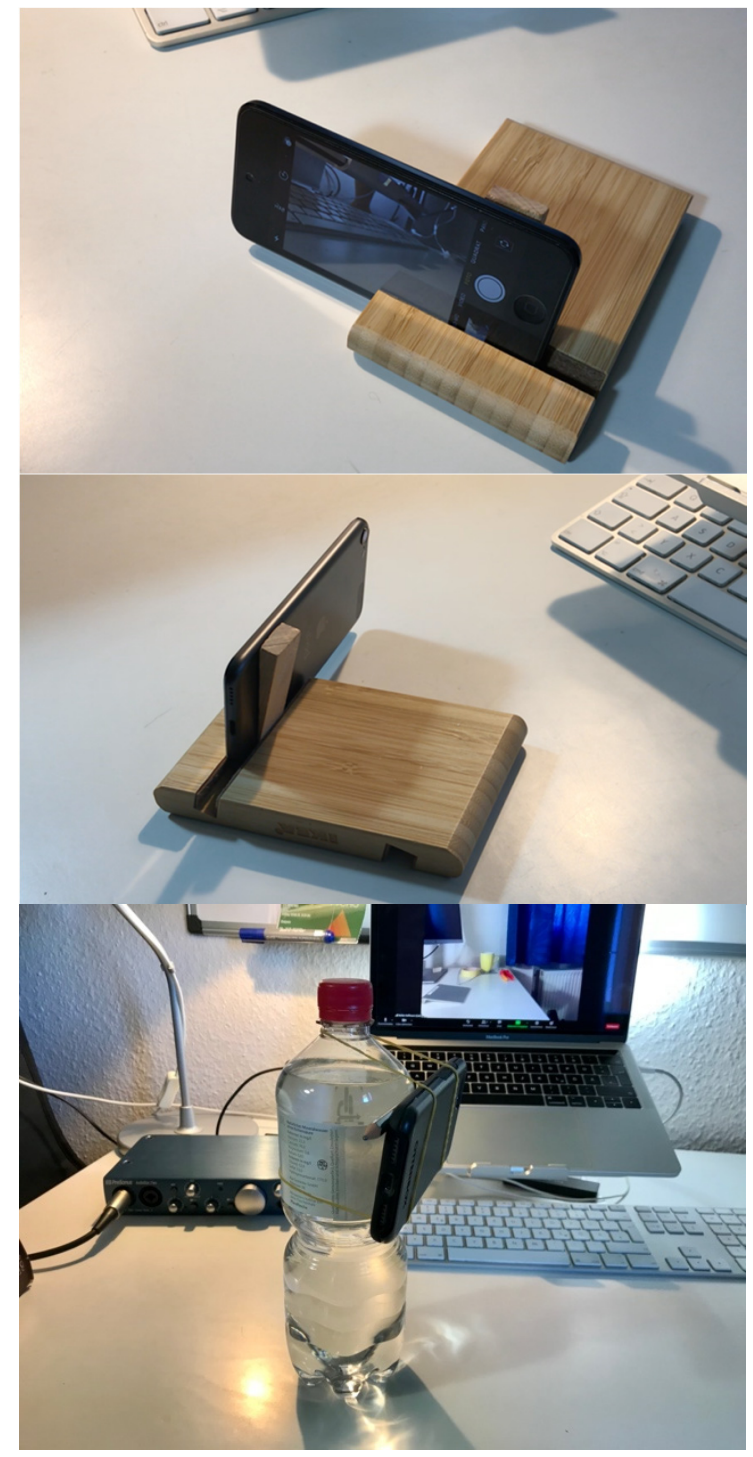

Abb. 6: Ausschnitt aus den Hinweisen für Klausurteilnehmer*innen mit kreativen Lösungsvorschlägen für die selbstgebaute Halterung eines Smartphones als Kamera. Weitere Ideen finden sich z.B. in YouTube Videos.

Classroom Format durchgeführt, mit durchwachsenen Ergebnissen.

Bei der Umstellung auf ein Online Format wird in BORs mit maximal 5 Personen gearbeitet. In jedem Raum ist ein Studierender als Tutor dafür verantwortlich, dass alle Aufgaben besprochen und alle Probleme geklärt werden. Hilfskräfte stehen als Mentoren bereit, um aufkommende Fragen zu beantworten und sonstige Hilfestellungen zu geben. Es zeigt sich, dass die Studierenden in einem solchen Format viel fokussierter arbeiten und der übliche Schwund in der Beteiligung im Laufe des Semesters deutlich geringer ist als in der Präsenzvariante. So hat an der Ab- 
schlussklausur ein deutlich höherer Prozentsatz der Studierenden teilgenommen als dies in der Präsenzvariante der Fall. Dies hatte keinen Einfluss auf die Bestehensquote, die sogar leicht besser war im Vergleich zu den vergangenen Jahren.

In einem Seminar über die Nutzung von Medien im Physikunterricht führen wir die Studierenden in die objektorientierte Programmierung mit Scratch [15] ein. Scratch ist eine kostenlose, für Kinder und Jugendliche leicht zugängliche Programmierumgebung, die auf der ganzen Welt verwendet wird. Die Studierenden erstellten in Gruppen einfache individuelle Programme in Scratch, um den Umgang mit dem Tool zu erlernen und die beim Programmieren verwendeten Objekte zu verstehen. Als Transferaufgabe wurde den Studierenden später im Semester der Roboter Codey Rocky (siehe Abb. 4) gezeigt, der mit mBlock5 programmiert werden kann. Die mBlock-Programmierumgebung unterstützt sowohl eine Scratch-basierte als auch eine Python-basierte Version von mBlock. Beide Programmiersprachen sind kostenlos über die mBlock-Website erhältlich [18]. Die Transferaufgabe beinhaltete eine ProgrammierChallenge, in der die Studierenden in Programmierteams gegeneinander antraten.

Die Programmier-Aufgabe wurde zunächst im Plenum der Veranstaltung vorgestellt (Abb. 7). In der restlichen Seminarsitzung konnten die Studierenden Teile Ihrer Programme in BORs des Zoom-Veranstaltungsmeetings ausprobieren, in dem sie die Programm-Dateien per Zoom-Chat an die Seminarbetreuer übermittelten. Die Betreuer spielten die Programme auf die im Labor vorhandenen Codey Rocky Roboter und führten die Programme live in ZOOM aus, so dass die Studierenden genaueres Feedback zur Wirkungsweise Ihrer Programmteile erhielten und somit zielgerichtet Änderungen am Code vornehmen konnten.

\section{Challenge:}

Schreiben Sie ein Programm, das die Folgen einer langen Sommerdürre für so viele Pflanzen wie möglich löst.

Gießen Sie die Pflanzen nach einem bestimmten Plan.

Dazu erhalten Sie die folgenden Informationen:

- Messdaten für die Bewegung des Codey Rocky's, die SuS für Sie aufgenommen haben

- Messdaten für die Betankung des Wasser-Reservoirs des Codey Rocky's, die SuS für Sie aufgenommen haben

- City Map:

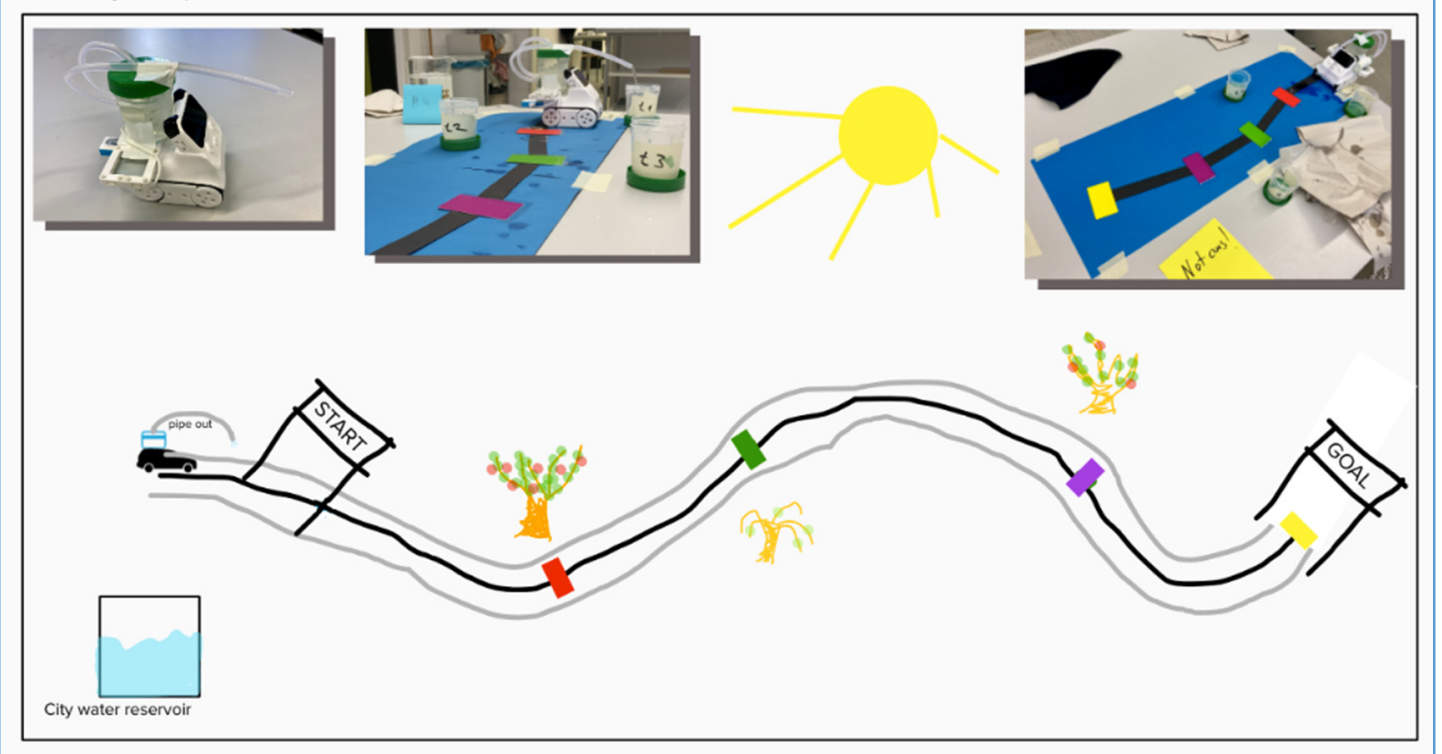

Abb. 7: Programmier-Challenge: Steckbrief der zu lösenden Aufgabe; zum Gießen modifizierter Codey-Rocky; Teststrecke; Durchführung der Challenge live in ZOOM. 
Basierend auf diesen Tests hatten die Teams zwei Wochen Zeit, die Challenge-Programme fertigzustellen und einzureichen. Die fünf fertigen Programme der Gruppen wurden vor der Seminarsitzung auf fünf verschiedene Codey Rockys aufgespielt und konnten live im ZoomVeranstaltungsmeeting gegeneinander antreten. Für den zweiten Teil des Semesters konnten sich einige Projektgruppen einen Codey Rocky und Materialien für die Erstellung ihres Projekts ausleihen.

\section{Was wird bleiben?}

Werden wir nach dem Ende der Pandemie wieder zu den alten Formen zurückkehren? Wir denken nicht, denn viele Kolleg*innen haben in der Krise neue Unterrichtsformen (inverted classroom etc.) und deren Vorteile für sich entdeckt! Dies wird sich sicher auch in der zukünftigen Präsenzlehre widerspiegeln. Vorlesungsaufzeichnungen sind gerade bei den Studierenden beliebt, da sie ein individuelles Lerntempo unterstützen. Dieses Tool wird uns erhalten bleiben, da auch an vielen Hochschulen aufgerüstet wurde, um die technischen Voraussetzungen dafür zu schaffen. Allerdings sollten die Dozenten darauf achten, dass nicht in jedem Jahr die alten Aufzeichnungen aus dem Schrank geholt werden, sondern die Veranstaltungen immer weiterentwickelt werden. Insgesamt erwarten wir, dass die Präsenzlehre weiter sehr wichtig bleibt, aber bewusster und zielgerichteter angewendet werden wird. Formate aus der Online-Lehre werden auch weiterhin eine Rolle spielen, aber in vielen Fällen eher als Ergänzung denn als Hauptunterrichtsform.

Aus unserer Sicht wird sich die Qualität der Lehre als Folge der Online-Lehre insgesamt verbessern! Dies liegt vor allem daran, dass sich viele Dozierende und Lehrer*innen mit neuen Lehrkonzepten auseinandersetzen mussten und deren Vorteile bei der konkreten Umsetzungen erfahren haben. Auf der anderen Seite hat die Online-Lehre aber auch gezeigt, wie wichtig soziale Interaktionen zwischen den Studierenden und mit den Lehrenden sind.

\section{Literatur}

[1] P. Greutmann: 19 Techniken des "Formative Assessment" (2019) in https://blog.hslu.ch/e-bausteine/fi les/2019/07/Greutmann Formatives-AssessmentTechniken-Allgemein Genehmigung.pdf

[2] https://www.mural.co

[3] https://www.lias.de/

[4] S. Carroll, S. Beyerlein, M. Ford, D.K. Apple: The Learning Assessment Journal as a tool for structured reflection in process education. Technology-Based ReEngineering Engineering Education Proceedings of Frontiers in Education FIE'96 26th Annual Conference, pp. 310-313 (1996)

[5] D. Maclsaac (SUNY Buffalo State College): persönliche Mitteilung

[6] G.M. Novak, E.T. Patterson, A.D. Garvin, W. Christian: Just-In-Time Teaching: Blending Active Learning With Web Technology (Prentice Hall Series in Educational Innovation, 1999)

[7] https://get.plickers.com

[8] https://kahoot.com

[9] C.H. Crouch, E. Mazur: Peer instruction: Ten years of experience and results. American Journal of Physics, 69(9), 970-977 (2001)

[10] https://phet.colorado.edu/sims/html/circuit-con struction-kit-dc/latest/circuit-construction-kitdc de.html

[11] https://phyphox.org/de/home-de/

[12] https://www.vernier.com/product/video-physicsfor-ios/

[13] https://phet.colorado.edu/

[14] https://education.makeblock.com/

[15] https://scratch.mit.edu/

[16] https://www.t-online.de/region/ko eln/news/id 89534636/koeln-verbotenes-team work-onlinepruefungen-erleichtern-betrug-an-deruni.html

[17] https://www.spiegel.de/panorama/bild ung/gerichtsentscheidungen-studenten-in-kiel-undhagen-muessen-online-ueberwachung-bei-prue fungen-dulden-a-ffdbfddd-c26f-421b-a6ff$788 \mathrm{cb} 8 \mathrm{f} 7 \mathrm{~d} 29 \mathrm{a}$

[18] http://www.mblock.cc/software/ 